\title{
405-nm pumped Ce3+-doped silica fiber for broadband fluorescence from cyan to red
}

\section{Amit Yadav, Nikolay B. Chichkov, Regina Gumenyuk, Evgeny Zherebtsov, Mikhail A. Melkumov, et al.}

Amit Yadav, Nikolay B. Chichkov, Regina Gumenyuk, Evgeny Zherebtsov, Mikhail A. Melkumov, Mikhail V. Yashkov, Evgeny M. Dianov, Edik U. Rafailov, "405-nm pumped Ce3+-doped silica fiber for broadband fluorescence from cyan to red," Proc. SPIE 10914, Optical Components and Materials XVI, 1091406 (27 February 2019); doi: 10.1117/12.2509599 


\title{
$405 \mathrm{~nm}$ pumped $\mathrm{Ce}^{3+}$-doped silica fiber for broadband fluorescence from cyan to red
}

\author{
Amit Yadav ${ }^{\mathrm{a}}$, Nikolay B. Chichkov ${ }^{\mathrm{a}}$, Regina Gumenyuk ${ }^{\mathrm{b}}$, Evgeny Zherebtsov ${ }^{\mathrm{a}}$, Mikhail. A. \\ Melkumov $^{\mathrm{c}}$, Mikhail V. Yashkov ${ }^{\mathrm{d}}$, Evgeny. M. Dianov ${ }^{\mathrm{c}}$, and Edik U. Rafailov ${ }^{\mathrm{a}}$ \\ ${ }^{a}$ Optoelectronics and Biomedical Photonics Group, AIPT, Aston University, Birmingham, B4 \\ $7 \mathrm{ET}, \mathrm{UK}$ \\ ${ }^{\mathrm{b}}$ Laboratory of Photonics, Tampere University of Technology, Tampere, Finland \\ ${ }^{c}$ Fiber Optics Research Center of the Russian Academy of Science, Moscow 119333, Russia \\ ${ }^{\mathrm{d}}$ Institute of Chemistry of High-Purity Substances of the Russian Academy of Sciences, Nizhny \\ Novgorod 603600, Russia
}

\begin{abstract}
A pure Ce-doped silica fiber is fabricated using modified chemical vapor deposition (MCVD) technique. Fluorescence characteristrics of a Ce-doped silica fiber are experimentally investigaed with continous wave pumping from $440 \mathrm{~nm}$ to $405 \mathrm{~nm}$. Best pump absorption and broad flourescence spectrum is observed for $\sim 405 \mathrm{~nm}$ laser. Next, the detailed anaysis of spectral response as a function of pump power and fiber length is performed. It is observed that a $-10 \mathrm{~dB}$ spectral width of $\sim 280 \mathrm{mn}$ can be easily achieved with different combinations of the fiber length and pump power. Lastly, we present, for the first time to the best of our knowledge, a broadband fluorescence spectrum with $-10 \mathrm{~dB}$ spectral width of $301 \mathrm{~nm}$, spanning from $\sim 517.36 \mathrm{~nm}$ to $\sim 818 \mathrm{~nm}$, from such fibers with non-UV pump lasers.
\end{abstract}

Keywords: Ce-doped fiber, broadband spectrum, rare earth doped, Ce-ion

\section{INTRODUCTION}

$\mathrm{Ce}^{3+}$-doped and $\mathrm{Ce}^{3+}-\mathrm{Ln}^{3+}$-codoped crystals, silicate, phosphate and fibers are of great interest for their broadband fluorescent emission across the visible and near IR wavelength range. $\mathrm{Ce}^{3+}$ ions have been investigated in aforementioned materials as independent luminescence centers and sensitizers. These Ce-doped materials has been used as scintillators and light emitters exploiting the allowed $5 \mathrm{~d}-4 \mathrm{f}$ transition in $\mathrm{Ce}^{3+}$ ions. ${ }^{1,2}$ On one hand radiation detection has been the motivation to forward the research on scintillators; ${ }^{3,4}$ and on the other hand Ce-doped silica fiber has been studied for non-invasive biomedical technique such as high resolution optical coherence tomography (OCT) ${ }^{5,6}$ At the same time, Ce has been used with phosphors to achieve white light from light-emitting diodes. ${ }^{7}$ However, due to excellent fluorescence spectrum promise of Ce-doped silica fibers they may be employed in future for both visible light emission and possibly supplement greatly exciting and rapidly growing field of visible light communication. Towards this the primary requirements for such fibers would be to ideally use non-UV pump sources and provide broadband emission spectrum across the visible part of the optical spectrum.

In this study, fluorescence properties of a Ce-doped silica fiber are experimentally investigated. The doped fiber is prepared using modified chemical vapor deposition (MCVD) technique. The fluorescence spectra of the doped fiber is examined under non-UV $405 \mathrm{~nm}$ continuous wave pump laser source. Fluorescence spectrum is observed to be strongly affected by the length of the fiber both in shape and peak emission wavelength. We also demonstrate, possibly for the first time, a broadband spectrum with a -10dB spectral width of $301 \mathrm{~nm}$ from such fibers.

Further author information: (Send correspondence to A.Yadav.)

A.Yadav.: E-mail:a.yadav1@aston.ac.uk

Optical Components and Materials XVI, edited by Shibin Jiang, Michel J. F. Digonnet,

Proc. of SPIE Vol. 10914, 1091406 - @ 2019 SPIE · CCC code: 0277-786X/19/\$18

doi: $10.1117 / 12.2509599$

Proc. of SPIE Vol. 10914 1091406-1 


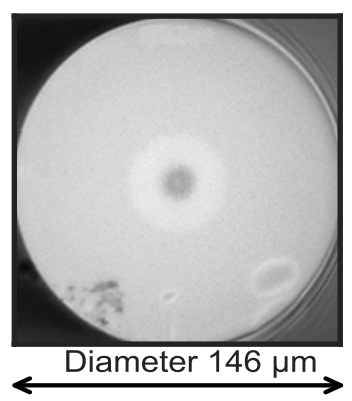

Figure 1. Cross-section of Ce-doped fiber.

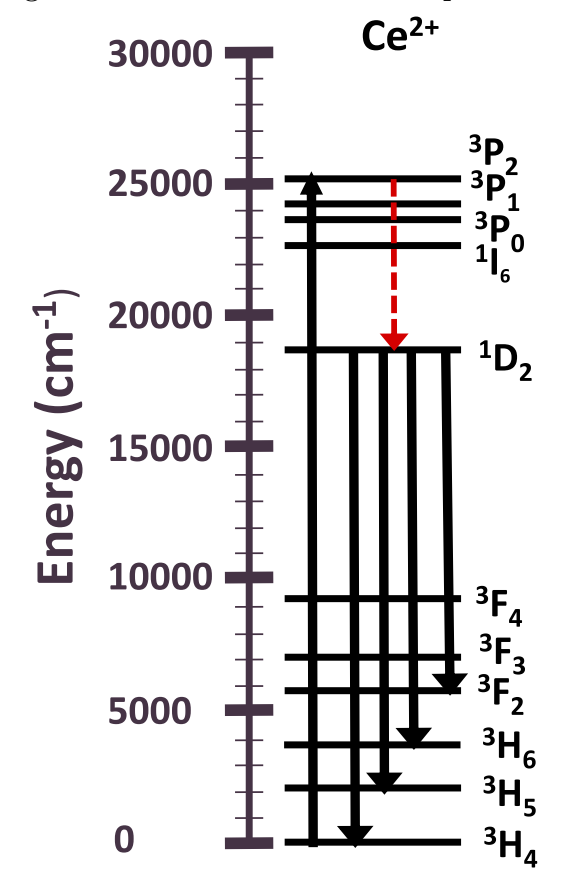

Figure 2. The proposed energy transfer mechanism for $\mathrm{Ce}^{2+}$ in Ce-doped fiber.

\section{EXPERIMENTAL DETAILS}

\subsection{Fabricating Cerium-doped fibers}

The Ce-doped silica cladding fiber is drawn from the preform fabricated by standard MCVD technology. The technological process of the preform fabrication consists of several principle steps. At the first step, the inner part of high purity silica substrate tube is deposited by powder like "soot" layer including $\mathrm{SiCl}_{4}$ and fluoride. This process is accompanied by simultaneous burning at the high temperature in the range of $1500-1700^{\circ} \mathrm{C}$. Introduction of fluoride ions allows to decrease the refractive index and form internal waveguide structure. At the next step, the rare-earth solution composed of $1 \mathrm{~mol} \%$ of $\mathrm{Ce}\left(\mathrm{NO}_{3}\right)_{3} \cdot 12 \mathrm{H}_{2} \mathrm{O}$ is introduced into the tube. The following sintering of the tube to evaporate the liquid from rare-earth dopant solution is carried at a temperature of $500^{\circ}$ C. Finally, the tube is collapsed and sealed. ${ }^{8}$ Figure 1 shows the cross-section of Ce-doped fiber. The fiber has near ideal circular shape with outer cladding and core diameters of $\approx 146 \mu \mathrm{m}$ and $\approx 13 \mu \mathrm{m}$, respectively.

\subsection{Characterization}

We suggest that the active fiber contains trivalent and divalent Ce ions. Upon absorbing blue light an electron in the trivalent Ce ions is excited from lower levels manifold of Ce ion $\left({ }^{2} \mathrm{~F}_{5 / 2}\right.$ and $\left.{ }^{2} \mathrm{~F}_{7 / 2}\right)$ to upper levels $\left({ }^{2} \mathrm{D}_{3 / 2}\right.$ and ${ }^{2} \mathrm{D}_{5 / 2}$ ). The electron can transit to the ground state of Ce ion emitting light in blue and green wavelength range. Due to the absence of allowed energy states between $4 \mathrm{f}-5 \mathrm{~d}$ orbital, the emitting properties of trivalent Ce 


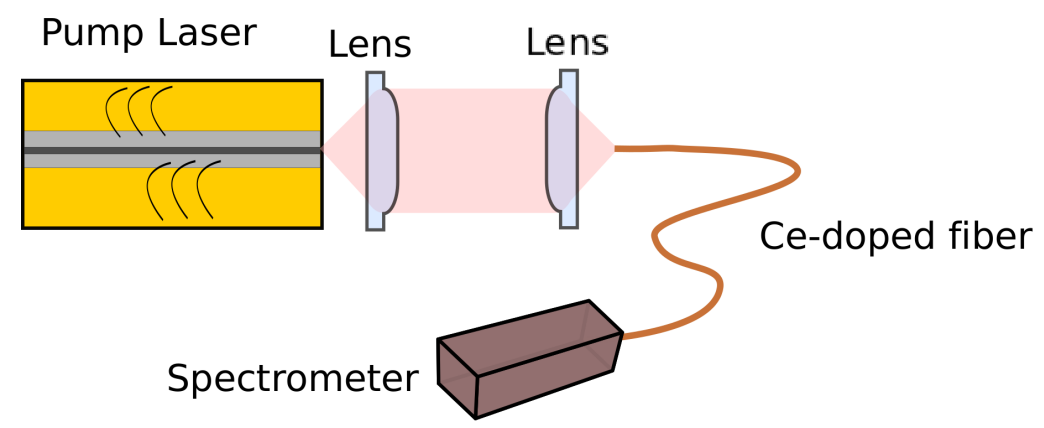

Figure 3. Experimental Setup.

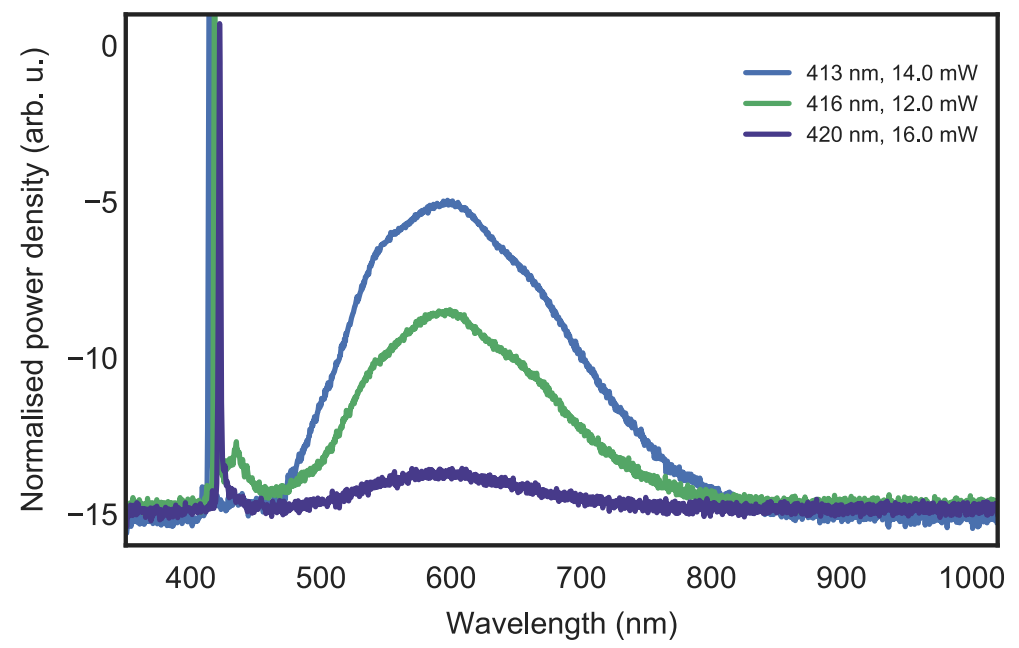

Figure 4. Fluorescence Spectrum at different pump wavelengths.

ion is limited in short part of the visible spectrum range. Therefore, $\mathrm{Ce}^{3+}$ ions mostly takes part in pump light absorption and emission/re-absorption of wavelength below $\approx 550 \mathrm{~nm}$, whereas, the luminescence properties of the fiber investigated in this article can be explained by down-conversion mechanism primarily occurred in $\mathrm{Ce}^{2+}$ sites. The Figure 2 shows the energy level diagram which is currently proposed to explain the intra-configuration transition of $\mathrm{Ce}^{2+}$ ions in the fiber. ${ }^{9}$ Divalent Ce characterizes by rich energy level structure with energy gap from 10000 till $25000 \mathrm{~cm}^{-1}$, which allows to cover all visible and short wavelength part of near-IR wavelength range. However, to efficiently exploit $\mathrm{Ce}^{2+}$ energy level structure it is necessary to minimize the parasitic non-radiative relaxation rate, which is high in pure silica glass host due to large phonon energy value $\approx 1100 \mathrm{~cm}^{-1}$. Therefore, the energy gap between upper excited and lower excited energy levels must be at least 4 phonons wide. As a consequence, any potential emitting levels in silica glass should be $4400 \mathrm{~cm}^{-1}$ above lower lying levels. ${ }^{10}$

To explore the quenching effect by multiphoton non-radiative relaxation and define the optimum energy transfer condition from the ground state to the upper excited states of $\mathrm{Ce}^{2+}$ ions, we experimentally investigate several pump sources with the optical excitation wavelengths of $440 \mathrm{~nm}, 420 \mathrm{~nm}, 416 \mathrm{~nm}, 414 \mathrm{~nm}, 413 \mathrm{~nm}, 405$ $\mathrm{nm}$. The excitation lasers used are TO-Can packaged diode lasers with continuous wave $(\mathrm{CW})$ output powers of $>5 \mathrm{~mW}$. The excitation lasers were temperature controlled at $20 \mathrm{degC}$ and their $\mathrm{CW}$ output is coupled to the fiber with a 1:1 A-coated aspheric lens assembly. The other end of the $\mathrm{Ce}^{3+}$-doped fiber is butt coupled to a multimode fiber, "QP-1-VIS-NIR", with core diameter of $600 \mu \mathrm{m}$. A schematic for the experimental setup is presented in Figure 3. The output of the multimode fiber is analyzed using a "Labsphere CDS-600" spectrometer and "SpectralSuite" software. The output optical power is also recorded at the far end of the multimode fiber. To maintain consistency, the output optical power and spectrum were measured alternatively in that order under the same excitation conditions. 


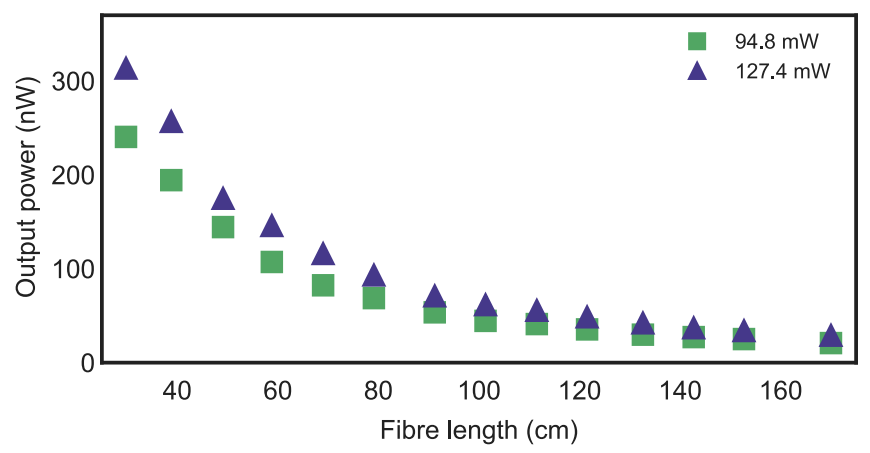

Figure 5. Fluorescence output power vs fiber length and pump power at $405 \mathrm{~nm}$.

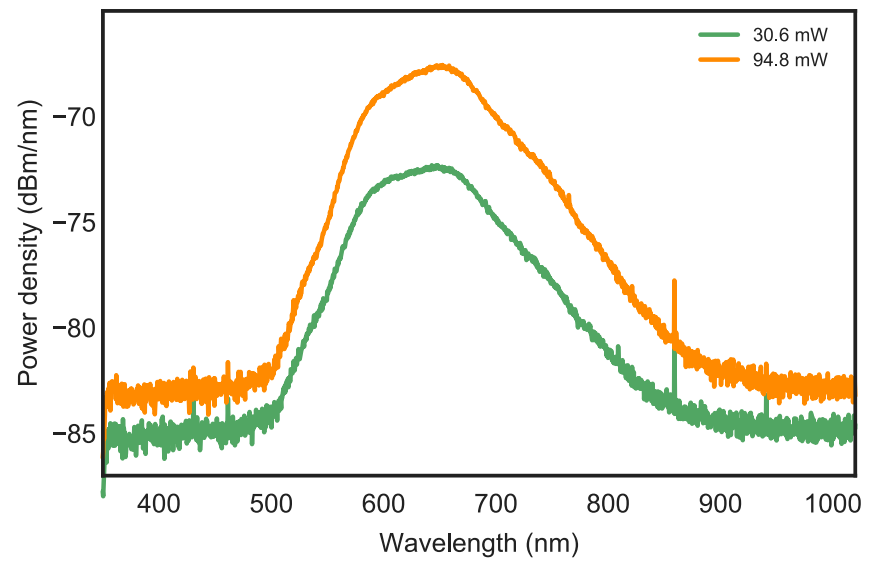

Figure 6. Measured spectra at different pump powers, obtained at fiber lengths of $132.6 \mathrm{~cm}(30.6 \mathrm{~mW})$, and $142.7 \mathrm{~cm}$ $(94.8 \mathrm{~mW})$

\section{RESULTS AND DISCUSSION}

To characterize fluorescence spectrum of ce-doped fibers it is first measured at different pump wavelengths, in the blue region of the optical spectrum, starting with $440 \mathrm{~nm}$ and moving towards shorter wavelengths. The fiber length used to identify the optimum pump wavelength is $\sim 70 \mathrm{~cm}$ and the pump power is kept between $12 \mathrm{~mW}$ to $18 \mathrm{~mW}$. Figure 4 depicts the fluorescence spectrum at selective pump wavelengths (refer to section 2.2). Following the trend on increased fluorescence with shorter pump wavelength it was observed that with $14 \mathrm{~mW}$ of $405 \mathrm{~nm}$ pump we observe the most efficient pump-to-signal wavelength conversion at $70 \mathrm{~cm}$ length of the fiber.

Next, the behavior of fluorescence spectra as a function of fiber length and pump power is investigated to determine the optimal parameters for broadband fluorescence. Initially spectral measurements for a $170 \mathrm{~cm}$ long fiber is performed at different discrete pump powers of $30 \mathrm{~mW}, 62.5 \mathrm{~mW}, 94.8 \mathrm{~mW}$ and $127.4 \mathrm{~mW}$. Spectral measurements at the same pump powers is then performed for different fiber lengths by slicing the fibers in the steps of $\approx 10 \mathrm{~cm}$ with the shortest fiber length of $30 \mathrm{~cm}$. The fiber is sliced from the end used for butt coupling as this allowed for consistency in recording output optical power at the far end of the ce-doped fiber due to large core diameter of the multimode fiber.

The measured fluorescence output power (excluding the residual pump at shorter fiber lengths) as a function of fiber length at different pump powers used is shown in Figure 5. The output power gradually increase with decrease of the fiber length reaching $>300 \mathrm{nW}$ for $\approx 30 \mathrm{~cm}$ of fiber length pumped with $>120 \mathrm{~mW}$ pump power.

Figure 6 depicts the observed fluorescence spectrum for two different fiber lengths at different pump powers. A correlation is observed between pump absorption and fiber length which is almost independent of applied pump power. The pump absorption is estimated from the measurement data to be $\sim 2 \mathrm{~dB} / \mathrm{cm}$. For fiber lengths $>58$ cm all pump is absorbed by the fiber, irrespective of optical pump power. It is also observed that fluorescence 

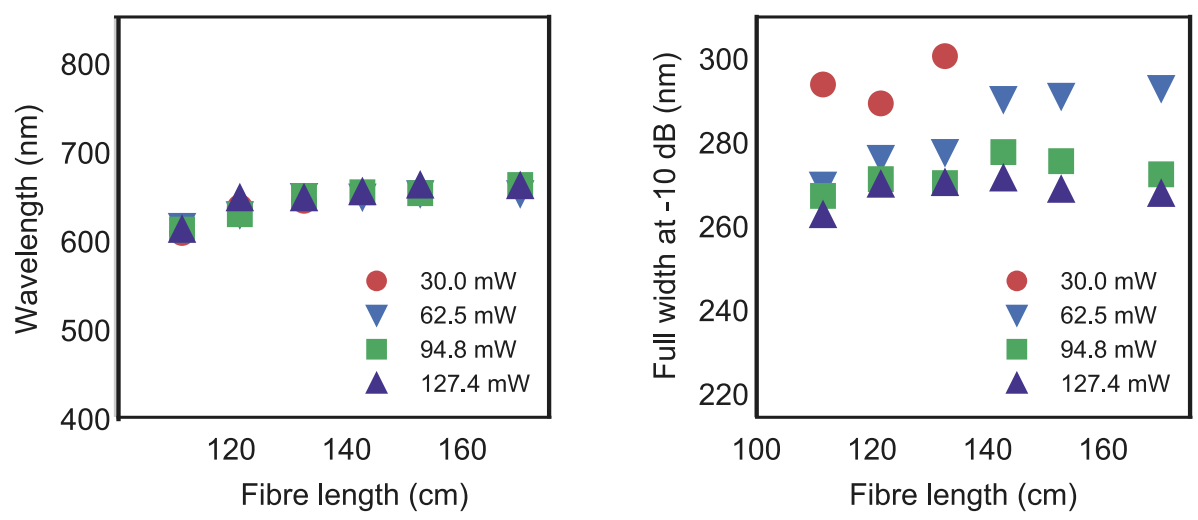

Figure 7. (a) Fluorescence bandwidth vs fiber length $(>100 \mathrm{~cm}$ ) and pump power: (a) peak wavelength (b) full-width at $-10 \mathrm{~dB}$.

spectrum shifts towards longer wavelengths with increasing fiber length and the spectral shape is also affected. The observed shift in peak emission wavelength is $>80 \mathrm{~nm}$ as a function of fiber length irrespective of pump power, see Figure 7a.

Furthermore, the fluorescence spectra due to $5 \mathrm{~d}-4 \mathrm{f}$ transitions on Ce-ions is broad as one would expect. We have observed that the spectral width over the whole range of fiber length and pump power used, increases almost linearly with increasing fiber length until a maximum is reached. The behavior has no observable correlation with pump power used. This $-10 \mathrm{~dB}$ spectral width is $>230 \mathrm{~nm}$. A -10 dB-bandwidth of $\sim 280 \mathrm{~nm}$ (see Figure $7 \mathrm{~b}$ ) is observed for different fiber lengths. A -10 dB-bandwidth of > $295 \mathrm{~nm}$ (see Figure 7b) is observed for fiber lengths of $\sim 132 \mathrm{~cm}$ To the best of our knowledge, this is the widest spectral bandwidth demonstrated from such fibers.

In conclusion, we demonstrate broadband, visible to near-IR, fluorescence from the pure Ce-doped silica fiber with $-10 \mathrm{~dB}$ spectral width of $>295 \mathrm{~nm}$ with peak wavelength $\sim 643 \mathrm{~nm}$ and peak power density of $0.06 \mathrm{nW} / \mathrm{nm}$.

\section{ACKNOWLEDGMENTS}

N. B. Chichkov thanks the Alexander von Humboldt Foundation for the personal support provided by the Feodor Lynen Research Fellowship. R. Gumenyuk is supported by Academy of Finland Postdoctoral research project (285170). E.Zherebstov is supported by Marie Skodowska-Curie grant agreement No 703145.

\section{REFERENCES}

[1] Vedda, A., Chiodini, N., Di Martino, D., Fasoli, M., Keffer, S., Lauria, A., Martini, M., Moretti, F., Spinolo, G., Nikl, M., Solovieva, N., and Brambilla, G., "Ce3+-doped fibers for remote radiation dosimetry," Applied Physics Letters 85, 6356-6358 (dec 2004).

[2] Jang, M. S., Choi, Y. H., Wu, S., Lim, T. G., and Yoo, J. S., "Material properties of the Ce3+-doped garnet phosphor for a white LED application," Journal of Information Display 17(3), 117-123.

[3] Chiodini, N., Fasoli, M., Martini, M., Morazzoni, F., Rosetta, E., Scotti, R., Spinolo, G., Vedda, A., Nikl, M., Solovieva, N., Baraldi, A., Capelletti, R., and Francini, R., "Rare-Earth Doped Sol-Gel Silicate Glasses for Scintillator Applications," Radiation Effects and Defects in Solids 158, 463-467 (jan 2003).

[4] Alshourbagy, M., Bigotta, S., Herbert, D., Guerra, A. D., Toncelli, A., and Tonelli, M., "Optical and scintillation properties of Ce3+ doped YAlO3 crystal fibers grown by $\mu$-pulling down technique," Journal of Crystal Growth 303(2), 500 - 505 (2007).

[5] Liu, C.-N., Huang, Y.-C., Lin, Y.-S., Wang, S.-Y., Huang, P.-L., Shih, T.-T., Huang, S.-L., and Cheng, W.H., "Fabrication and Characteristics of Ce-Doped Fiber for High-Resolution OCT Source," IEEE Photonics Technology Letters 26, 1499-1502 (aug 2014). 
[6] Liu, C.-N., Huang, Y.-C., Huang, P.-L., Chen, N.-K., Yu, C.-P., Huang, S.-L., and Cheng, W.-H., "Broadband Ce/Cr-doped crystal fibers for high axial resolution OCT light source," Optics Express 23, 29723 (nov 2015).

[7] Hong, Y., Jinlei, C., Yong, P., Tiejun, Z., and Shucai, G., "Photoluminescence properties of Tb3+ and Ce3+ co-doped Sr2MgSi2O7 phosphors for solid-state lighting," Journal of Rare Earths 33(4), 366-370 (2015).

[8] Khopin, V. F., Umnikov, A. A., Guryanov, A. N., Bubnov, M. M., Senatorov, A. K., and Dianov, E. M., "Doping of optical fiber preforms via porous silica layer infiltration with salt solutions," Inorganic Materials 41(3), 303-307 (2005).

[9] Ma, C.-G., Brik, M. G., Liu, D.-X., Feng, B., Tian, Y., and Suchocki, A., "Energy level schemes of fN electronic configurations for the di-, tri-, and tetravalent lanthanides and actinides in a free state," Journal of Luminescence 170, 369-374 (2016).

[10] Dejneka, M. J., Streltsov, A., Pal, S., Frutos, A. G., Powell, C. L., Yost, K., Yuen, P. K., Müller, U., and Lahiri, J., "Rare earth-doped glass microbarcodes," Proceedings of the National Academy of Sciences 100(2), 389-393 (2003).

[11] Atkins, R. M., "Measurement of the ultraviolet absorption spectrum of optical fibers," Optics Letters 17(7), 469-471 (1992).

[12] Xu, X., Lebbou, K., Moretti, F., Pauwels, K., Lecoq, P., Auffray, E., and Dujardin, C., "Ce-doped LuAG single-crystal fibers grown from the melt for high-energy physics," Acta Materialia 67, 232-238 (apr 2014). 